\title{
Estimation Of Fuzzy Structure Parameters For Continuous Junctions
}

\author{
C. Soize ${ }^{1}$ and K. Bjaoui ${ }^{2}$ \\ ${ }^{1}$ Structural Dynamics and Coupled Systems Department, ONERA, BP 72, 92322 Chatillon Cedex, France. \\ 2 GAUS, Département de Génie Mécanique, Sherbrooke (Qc), Canada, J1K 2R1.
}

\section{Introduction}

The fuzzy structure theory was introduced by Soize [1] 15 years ago in order to model structural complexity in the medium frequency range. This structural complexity plays a fundamental role in the response of a master structure coupled with complex substructures in the context of structural-acoustic systems. In 1993, a second fuzzy impedance law was proposed [2] to model the case of fuzzy substructures attached to the master structure through a continuous junction. Since 1993, much research has been conducted concerning the problem of a master structure coupled with a large number of simple linear oscillators but few concerning continuous cases. Two main problems had to be solved to be able to apply the fuzzy structure theory (described in details in Ref. [3]) to the case of continuous junctions. The first problem was related to the construction of a procedure for identifying the fuzzy structure parameters in order to solve complex problems and to allow experimental identifications to be performed. The second one, which requires solving the first, is related to validation of the fuzzy structure theory for continuous junctions. A general procedure has been developed to solve the first problem. In this paper, we present a first validation of the fuzzy structure theory for continuous junctions between the master structure and the complex substructures. We introduce a new cost function replacing the cost function previously introduced [4]. It allows an efficient estimation of the fuzzy structure parameters using a procedure based on the statistical energy approach (SEA).

\section{Reference complex structure}

The reference complex structure is made of a master structure coupled with four complex substructures (a), (b), (c) and (d) (see Figure 1). The master structure is made of two rectangular homogenous isotropic thin plates (1) and (2), in bending modes and simply supported, coupled along their common edge; the rotation around this edge is continuous. Each complex substructure is made of a rectangular homogenous isotropic thin plate on which many simple oscillators are attached. The plate of each complex substructure is in bending modes and simply supported, coupled to a plate of the master structure along their common edge; the rotation around this edge is continuous. Consequently, there is a continuous junction between the master structure and each complex substructure. Two plates belonging to different complex substructures are not coupled along their common edge. The method used to construct the model of the reference complex structure consists in constructing the generalized impedance matrix of an isolated plate belonging to the master structure and an isolated complex substructure. Then the coupling between the isolated subsystems is done using a Lagrange multiplier technique in order to express the continuity of the rotation on the junctions.
A dynamical response of the reference complex structure is calculated for a force excitation applied at a point of a master structure plate. It should be noted that at low frequency range the complex substructures do not significantly affect the response of the master structure. In medium frequency range, the complex substructures play an important role in the response of the master structure; complex substructures induce an apparent strong damping in the master structure due to the power flow from the master structure to structural complexity.

\section{Reference complex structure modeling using the fuzzy structure theory}

Using the fuzzy structure theory, the reference complex substructure is modeled by a fuzzy structure. The effects of each fuzzy substructure on its related master structure plate are taken into account using a random boundary impedance (on $\Gamma_{(1), \text { fuz }}^{(\text {a) }}, \Gamma_{(1), \text { fuz }}^{(\text {b) }}$ for plate (1) and on $\Gamma_{(2), \text { fuz }}^{(\text {c) }}, \Gamma_{(2), \text { fux }}^{(\text {d) }}$ for plate (2)) whose expression can be found in Ref. [5-6]. For each fuzzy substructure, the impedance law related to each substructure depends on four mean coefficients (parameters) and their associated deviation coefficients. These mean parameters are the mean coefficient of the participating inertial moment, the mean rate of internal damping, the mean modal density and the mean equivalent coupling factor. The generalized impedance matrix of a master structure plate coupled with its fuzzy substructure is constructed using Ritz-Galerkin method. The coupling between the two plates of the master structure is written using Lagrange multipliers technique as done before. The construction of the random response of the fuzzy structure is based on the use of Neumann series expansion limited to order 2 . Then, we obtain the mean response function of the fuzzy structure. And in order to get more information about the random response level in $\mathrm{dB}$, we construct the confidence region defined by the upper and lower envelopes of the frequency-response-function modulus corresponding to a given probability level. The upper envelope is constructed using Chebychev's inequality.

\section{Estimation of fuzzy structure mean parameters}

It is assumed that a direct estimation of the mean rate of the internal damping and the mean modal density of the fuzzy substructures can be obtained. Since the junction between a plate of the master structure and one of its fuzzy substructures is continuous, the fuzzy structure theory yields a mean equivalent coupling factor much lower than 1. A sensitivity analysis was performed and showed that the frequency independent value 0.005 was a good approximation for this mean parameter. 
Concerning the estimation of the mean coefficient of the participating inertial moment, we use the statistical energy approach introduced in [2], but using a new cost function [5-6]. The mean coefficient of the participating inertial moment is obtained minimizing this new function cost. But this cost function is not convex, then the optimization problem is not easy to solve. Consequently, we developed an algorithm with two main steps. In the first step, a neighborhood containing the solution of the optimization problem is constructed using a random research algorithm. In the second step, the optimization problem is solved in this neighborhood.

\section{Validation}

We consider the response of the reference complex structure and the mean response of the fuzzy structure with zero deviation coefficients. It was shown that the fuzzy structure mean response with zero deviation coefficients gives a good representation of the reference complex structure response.

We consider the response of the reference complex structure and the envelopes of the fuzzy structure response with nonzero deviation coefficients. The deviation coefficients associated with the participating inertial moments and the modal densities are nonzero whereas those associated with internal damping rates and equivalent coupling factors are taken equal to zero. It was shown that the response of the reference complex structure belong to the confidence region defined by the upper and lower envelopes predicted by the fuzzy structure theory and corresponding to a probability level equal to 0.95 . Consequently, the prediction is satisfactory and this example validates the fuzzy structure theory for continuous junctions.

\section{Conclusion}

This paper constitutes a first validation of the fuzzy structure theory for continuous junctions between the master structure and the fuzzy substructures. The mean response function calculated by the fuzzy structure theory with zero deviation coefficients gives a good representation of the reference complex structure response. The calculation of the envelopes with nonzero deviation coefficients based on the use of a second-order Neumann series expansion and Chebychev's inequality is very efficient. In addition, a new function cost allowing estimation of fuzzy structure parameters has been introduced. Statistical energy approach has been proved very efficient This procedure for estimating the fuzzy structure parameters, validated by numerical simulation, opens the field to experimental identifications.

\section{References}

[1] Soize, C., "Probabilistic structural modeling in linear dynamic analysis of complex mechanical systems. ITheoretical elements", Rech. Aerosp. 5, pp. 23-48, (1986). (English edition).

[2] Soize, C., "A model and numerical method in the medium frequency range for vibroacoustic predictions using the theory of structural fuzzy", J. Acoust. Soc. Am. 94, pp. 849-865, (1993).

[3] Ohayon, R. and Soize, C., "Structural Acoustics and Vibration", Academic, San Diego, (1998).

[4] Soize, C., "Estimation of the fuzzy substructure model parameters using the mean power flow equation of the fuzzy structure", Proceeding of the ASME Noise Control and Acoustics Divisions, Vol. 1, ASME/WAM, 28-30, NCA-Vol. 22, pp. 23-30 (1996), also published in Journal of Vibration and Acoustic 120, pp. 279-286 (1998).

[5] Bjaoui, K., "Estimation des paramètres d'une structure floue pour des jonctions continues", Thèse du Conservatoire National des Arts et Métiers, Paris, (1999).

[6] Soize, C. and Bjaoui, K., "Estimation of fuzzy structure parameters for continuous junctions", J. Acoust. Soc. Am. 107 (4), pp. 201 1-2020, (2000).
Fuzzy

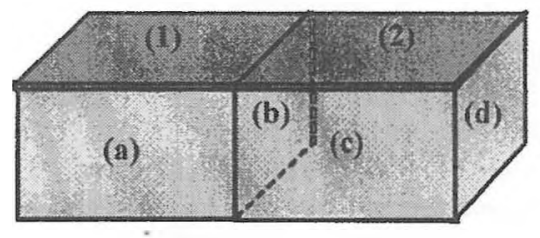

Complex structure structure

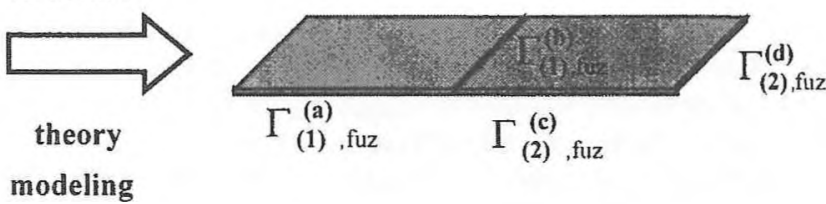

Fuzzy structure

Fig. 1. Model of the reference complex structure by fuzzy structure theory 